\title{
Unmaking: Enabling and Celebrating the Creative Material of Failure, Destruction, Decay, and Deformation
}

\author{
Katherine W. Song \\ kwsong@berkeley.edu \\ University of California, Berkeley \\ Berkeley, California
}

\author{
Eric Paulos \\ paulos@berkeley.edu \\ University of California, Berkeley \\ Berkeley, California
}

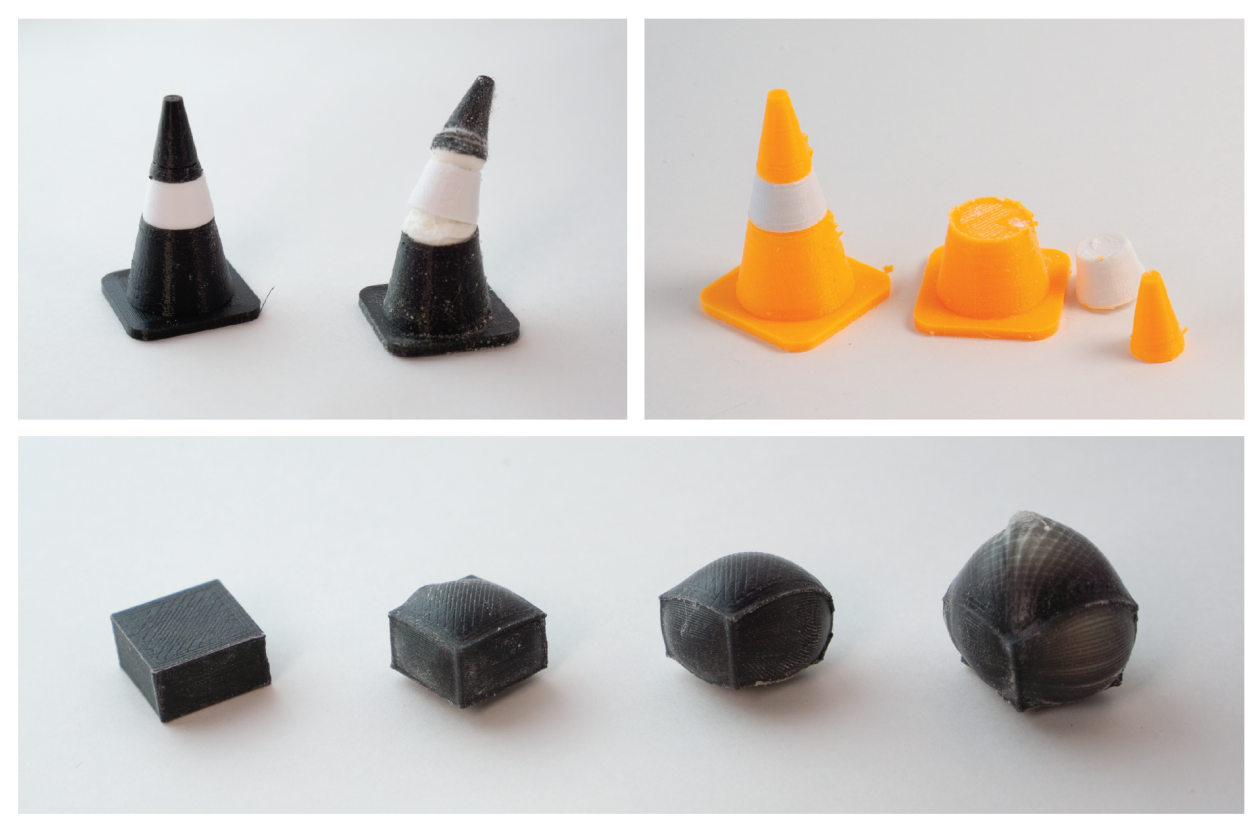

Figure 1: Exemplar unmaking designs achieved using multi-material 3D printing, exhibiting splitting (top left), breaking (top right), and bulging (bottom)

\begin{abstract}
The access and growing ubiquity of digital fabrication has ushered in a celebration of creativity and "making." However, the focus is often on the resulting static artifact or the creative process and tools to design it. We envision a post-making process that extends past these final static objects - not just in their making but in their "unmaking." By drawing from artistic movements such as Auto-Destructive Art, intentionally inverting well-established engineering principles of structurally sound designs, and safely misusing unstable materials, we demonstrate an important extension to making - unmaking. In this paper, we provide designers with a new vocabulary of unmaking operations within standard 3D modeling tools. We demonstrate how such designs can be realized using a novel multi-material 3D printing process. Finally, we detail how unmaking allows designs
\end{abstract}

This work is licensed under a Creative Commons Attribution-Share Alike International 4.0 License.

CHI '21, May 8-13, 2021, Yokohama, Japan

(c) 2021 Copyright held by the owner/author(s).

ACM ISBN 978-1-4503-8096-6/21/05.

https://doi.org/10.1145/3411764.3445529 to change over time, is an ally to sustainability and re-usability, and captures themes of "aura," emotionality, and personalization.

\section{CCS CONCEPTS}

- Human-centered computing $\rightarrow$ Human computer interaction (HCI); Interactive systems and tools.

\section{KEYWORDS}

digital fabrication, making, unmaking, sustainability, destruction, interaction design, temporality, materiality, art

\section{ACM Reference Format:}

Katherine W. Song and Eric Paulos. 2021. Unmaking: Enabling and Celebrating the Creative Material of Failure, Destruction, Decay, and Deformation. In CHI Conference on Human Factors in Computing Systems (CHI '21), May 8-13, 2021, Yokohama, Japan. ACM, New York, NY, USA, 12 pages. https://doi.org/10.1145/3411764.3445529

\section{INTRODUCTION}

While the dominant western narrative of beauty when it comes to describing physical artifacts is largely one of strength, durability, and perfect proportions, the very opposite - fragility, impermanence, and "imperfection" - has long been embraced across 
multiple cultures as uniquely beautiful and meaningful [47]. One of the most well-known examples of this is wabi-sabi, an ancient Japanese aesthetic rooted in Zen Buddhist philosophy that honors the beauty and authenticity of what is impermanent, incomplete, and conventionally imperfect [24]. Throughout the years, artistic movements around the world have resonated with these concepts. For instance, in 1959, Gustav Metzger founded the Auto-Destructive Art movement as a reaction to the repulsiveness of the violence and destruction witnessed during World War II [35]. Inspired by earlier avant-garde movements like Cubism and Dadism that rejected the notion of mainstream societal values and aesthetics, Metzger argued that by allowing and forcing their art to disintegrate, artists could create powerful controversies that were a "kind of mass-therapy" - bringing destruction into consciousness and forcing the public to reckon with it - and an "educational programme" - allowing artists and spectators to form more intimate relationships with the materiality and temporality of the art. Metzger and his contemporaries successfully created public Auto-Destructive Art with acid, fire, and machines, but Metzger acknowledged that his theory was decades ahead of being able to be fully realized in practice, explicitly calling for collaboration between artists and engineers to create tools to more easily generate art that would disintegrate. While artists have remained interested in and still create art echoing Metzger's manifestos (Man Ray's Object to Be Destroyed (1923), Jean Tinguely's Homage to New York (1960), Niki de Saint Phalle's Shooting Picture (1961), Yoko Ono's Cut Piece (1964), John Baldessari's The Cremation Project (1970), Chris Burden's Samson (1985), and Banksy's Girl With Balloon (2018), to name a few), the technological tools that Metzger envisioned arguably have not yet been fully realized.

This paper responds to Metzger's call, leveraging new creative materials enabled through recent advances in technology, design, Human-Computer Interaction (HCI), and digital fabrication. As an extension to the focus on making that has characterized digital fabrication research, we propose unmaking - our term to encapsulate the processes of destruction and decay that can and should be equally considered as part of the creative making process. Among other values that we detail in this paper, designing for unmaking creates a unique opportunity for the designer of an object, the user, and the environment to collaboratively create "aura," a concept of uniqueness and authenticity that Walter Benjamin, perhaps prematurely, lamented the loss of in the ushering of our current "age of mechanical reproducton" [3].

Structuring our paper accordingly, we summarize our main contributions as follows:

- Introduction of unmaking as an under-explored design opportunity that expands the creative process of physical design

- Framing of the foregrounding considerations of sustainability and re-usability within the field of digital fabrication through unmaking

- An unmaking vocabulary that can be operationalized within a digital fabrication - particularly 3D-printing - framework

- Demonstration of a workflow that allows makers to design and fabricate 3D-printed objects that exhibit controllable unmaking aesthetics
As the first introduction of our concepts, we focus our examples and discussion mainly around 3D printing, an important and popular digital fabrication technology that boasts portability, low-cost options, and ease of integration with multiple materials. However, unmaking as a concept can absolutely be applied to other digital fabrication techniques as well, a point that we address in our Discussion.

\section{RELATED WORK}

\subsection{Destruction as an Aesthetic}

Drawing inspiration from wabi-sabi and similar earlier philosophy, the HCI community has mused upon the artistic value that signs of destruction, such as visible damage and wear, might add to physical artifacts in recent years [49, 50]. For instance, in 2014, Ikemiya and Rosner presented Broken Probes, a study of the reassembly of shattered objects in a style reminiscent of kintsugi, the Japanese art of repair that highlights cracks with gold or decorative lacquer instead of concealing them [19]. Their user study suggested that reassembly gave new life to the objects, embodying narratives, acceptance of loss, and more generally the concept of time. Similarly, Zoran and Buechley embraced the idea that the signs of an object's breakage should be memorialized for its unique artisanship and story, utilizing digital fabrication techniques to reassemble broken objects with a new, hybrid aesthetic [62]. Design explorations in deliberately-created patina or "material traces" of wear have also suggested that such aesthetics are perceived favorably as signs of maturation and ways to add personal value to physical objects [16].

Destruction occurring over a longer span of time due to environmental exposure - described most often as degradation or decay in literature - has also been celebrated as an aesthetic that is unique to biological materials, especially in more recent years as researchers have sought to motivate the use of environmentally friendly media [29-31]. Along with embodying the sentiments and values already expressed, the degradation of biological materials provides novelty and also forces us to reflect upon and consciously deepen our relationship with nature.

\subsection{Destruction as a Creative Act}

In addition to the added aesthetic value that destruction can bring, existing literature suggests that the act of destruction itself can be entertaining, empowering, informative, and cathartic. Devendorf and Rosner's 3D Print Eraser and Melt are speculative systems in which digital fabrication machines are used to erase or induce the destruction of material objects, with the main purpose being to create provocative performance art [11]. Other scholars have further suggested that enabling human interaction in processes related to destruction can develop additional meaning and value. Through a series of several detailed inquiries, Murer et al. proposed "uncrafting," or the hands-on disassembly of interactive artifacts that could be considered a methodical or controlled form of "destruction," as a means to gain material understanding and to inspire future designs [36-39]. Also noteworthy is the concept of "counterfunctional design," a design methodology proposed by Pierce and Paulos to draw attention to and force the rethinking of certain elements of a design by "destroying" the concept of the conventional function of a device and instead creating inhibiting interfaces [43]. In other 
demonstrations, fabrication machines augment humans' abilities as agents of destruction. For example, Eickhoff et al.'s Destructive Games re-purpose fabrication machines as tools to enable games that resulted in the destruction of physical objects, such as money bills and toys, and the authors were surprised to report that 8 out of 12 of the participants in their user study said that they would play such games again [15].

Perhaps the findings of Destructive Games should not be so surprising, however. In 2012, Ringler and Reckter conducted an inquiry into whether humans would be tempted to destroy their robot and similarly found that the majority of their users chose to cause obvious damage to the robot and reported some sense of satisfaction in doing so, despite feeling that their actions were cruel [44]. The worldwide popularity of "Rage Rooms" - rooms filled with objects that individuals or groups pay to wreck - is a testament to what may be our inherent desire to destroy [34]. Indeed, despite its negative connotations, destruction is a central concept in psychotherapy for letting go and coping with loss, and several scholars have argued that we ought to embrace and enable the embodiment of these emotional needs in design $[32,45]$.

\subsection{Role of 3D Printing in Destruction}

The work that we have offered so far suggests that the argument that embodying destruction, decay, and deformation can bring unique meaning to design is itself not a new one in HCI. However, from existing literature alone, it remains unclear how to achieve or design this in a practical sense. Thus far in this paper, we have presented prior work that uses digital fabrication machines to reassemble broken pieces [62], actively destroy objects [11, 15], and print biological materials that easily degrade [29]. Still, the possibility of digitally designing and fabricating objects that unmake in controllable or pre-defined ways post-making has been largely unexplored.

The approach that we take in this paper is to leverage the power of multi-material 3D printing. For this, the findings in the world of $4 \mathrm{D}$ printing and shape-changing interfaces provide valuable techniques and inspiration. $4 \mathrm{D}$ printing is the process by which objects are fabricated in 2 or 3 dimensions but, upon exposure to a catalyst or stimulus, can change shape, color, or other material properties in a controllable manner [27, 48, 57]. The promise to help solve numerous design problems in transportation, architecture, assembly, and other applications has driven the rapid development and characterization of new materials and $4 \mathrm{D}$ printing techniques. One common approach for shape-shifting in particular is to take advantage of the shape-memory properties of conventional thermoplastics used in 3D printing by selectively building up stress during printing with print speed and direction. Upon heating, this stress is released in the form of a pre-determined shape change $[1,14,17,18,26,51,55,56]$. Several researchers have also use the approach of turning to unusual materials, such as foaming agents [20] and living organisms [60], to induce complex changes in not only shape but texture, color, and other visible or tangible properties. Many other active investigations in novel "smart" materials from the field of materials science may eventually prove to be fruitful to draw upon as well $[5,9,10,27,46,57]$.
This is not to say, however, that unmaking is a subset of 4D printing, as the two have distinct goals. Upholding structural integrity is a theme that is prevalent throughout $4 \mathrm{D}$ printing literature, but it is one that unmaking challenges. Nonetheless, reading such literature through a different lens may reveal great insights for unmaking with 3D printing strategies. For example, Gu et al. present Geodesy as a $4 \mathrm{D}$ printing method that results in controllable shape-shifting, but they specifically avoid deformations that result in strength reduction [18]. We believe that Gu's approach of building in heatactivated shrinkage zones can be leveraged for unmaking, but to do this, we shall embrace, not avoid, instability.

\section{UNMAKING AND SUSTAINABILITY}

Sustainability is an increasingly pressing global concern that demands particular attention when discussing energy and materialintensive efforts, including physical prototyping. Sustainability also has deep roots in HCI research. We want to respect and acknowledge the importance of environmental considerations when proposing new frameworks for designing with physical materials. As such, before presenting our vocabulary and workflow for unmaking, we dedicate this separate section to reflecting upon how unmaking can be an ally in the movement towards more sustainable thinking and practices.

\subsection{Sustainable "Making"}

Several scholars have proposed guidelines for developing and assessing sustainability practices in digital fabrication and design $[4,6,13,25,33,42]$. When considering what materials to use, Lazaro Vasquez, Wang, and Vega urge researchers to use Life Cycle Analysis to assess the sustainability of their practices; in addition to taking into account the energy consumed by machines themselves, researchers should choose materials with the lowest "embodied energy" and carbon dioxide emissions possible and recycle or dispose of materials appropriately [25]. There have been several successful and provocative demonstrations of artifacts and workflows embodying these principles with easily degradable, biological materials, such as mycelium [21, 52, 53, 58], but overall, the field has been slow to change. As previously discussed, the unique aesthetic of biological materials has been highlighted and celebrated. However, according to Lazaro Vasquez, Wang, and Vega's review of the environmental impact of physical prototyping, over one-third of physical prototyping reported in the last 5 years of $\mathrm{CHI}$ proceedings was still done with plastics, suggesting that more needs to be done to make the case for easily degradable materials [54]. Beyond simply appealing to makers' sense of environmental responsibility, bio-degradable and compostable materials offer arguably few advantages. PLA, which alone accounts for $25 \%$ of the prototypes Lazaro Vasquez et al. surveyed, is touted as a eco-friendly material that can compost in just 90 days. Unfortunately, in practice, much of it ends up in the landfill, where it can take up to 1000 years to decompose, because composting PLA requires specific industrial conditions that are not always accessible in a given locale. This should not be particularly unexpected, as the development of such materials for prototyping is currently driven by the perceived need to meet the same durability and mechanical properties of their conventional counterparts, inherently limiting their ability to degrade 
- certainly in the consumer's hands but even when considering industrial capabilities.

Grappling with the waste that results from current practices is a major challenge that plagues digital fabrication and maker culture [12, 23]. In 2007, Eli Blevis laid out several principles for design from a sustainability perspective, demanding that invention be "linked" with disposal - that is, invention should not be made without a detailed plan for the disposal of materials that will result - and that renewal and reuse be prioritized [4]. Several efforts have certainly been made regarding the latter. As part of their framework of "salvage fabrication" and bettering practices around sustainability in the makerspace, Dew et al. imagined that makers might be interested in concept of "perishable printing" - working with digital fabrication materials that are designed to decay - to raise awareness around the waste and leftovers of 3D printing [12]. At CHI 2020, Wu and Devendorf made critical strides towards eliminating waste in the realm of smart textiles altogether, presenting Unfabricate, an inquiry into designing smart textiles designed with disassembly and reuse as a focus [59].

\subsection{Sustainable "Unmaking"}

Continuing with Blevis's framework, we envision unmaking playing a critical role in promoting renewal and reuse. For materials such as PLA that are bio-based but not consumer-degradable, mechanical recycling - shredding the material into small parts to be remelted and renewed - can be energy-intensive, but it can in some cases be lower in environmental impact than composting when considering the entire life cycle of the material [8]. Aesthetics aside, creating objects that can spontaneously shred, or at least disassemble into their constituent colors and materials, is one practical way in which a designer can use unmaking to facilitate renewal and reuse.

Additionally, unmaking finds unique relevance in addressing Blevis's call to "link invention and disposal," which has proven somewhat more elusive than the promotion of renewal and reuse. By capturing the values surrounding ideas of destruction that have previously been identified, unmaking radically counters the conventional concepts of disposal and waste themselves, turning the process of disposal into one of continual invention. Our vision is that drawing researchers to the space of unmaking will further sustainability agendas, incentivizing the search and development of novel materials designed specially for unmaking - ones that can be degraded by an individual upon demand without reliance on energy-intensive processes and are "eco-friendly" in a truer sense than materials available today.

Finally, while sustainability is an important theme that is enabled and supported by unmaking, we argue that unmaking as a concept itself has valuable design, aesthetic, and philosophical implications alone, even within the limitations of currently available materials.

\section{THE DESIGN VOCABULARY OF UNMAKING}

Once one accepts that designing for unmaking is a worthwhile pursuit, it is natural to wonder what the space of unmaking actually looks like and how to harness known digital fabrication materials and tools. Currently, in the world of three-dimensional modeling, software design tools offer atomic operations such as extrude, loft,
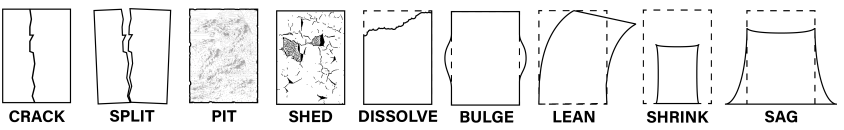

Figure 2: Visual icons representing selected elements of the unmaking design language

and revolve. As more practitioners begin to embrace unmaking within their designs, a new set of "unmaking" operations will be needed. The power of unmaking is not in a naive view of reckless destruction but in the poetic way in which a designer creates and layers the unmaking experience within an object.

We propose an initial set of expanded design vocabulary for unmaking, drawing upon aesthetics expressed in previously-discussed art and HCI research. First, from wabi-sabi-inspired research [19, 50, 62] or "un-crafting" work [36-39] in which the destruction aesthetic is characterized by clean, cleavage lines, we propose the inclusion of crack and split operations. Other work has emphasized the value of other visible signs of aging and use, such as surface nicks or rusting [16, 30,31]; for these, we offer pit and shed unmaking operations. Biological materials, which are especially attractive choices for sustainable unmaking, tend to destruct by dissociation or disintegration [29], so we propose that the dissolve operation be included to capture this aesthetic. Biological and water-based materials are also susceptible to warpage in response to environmental factors such as temperature and humidity [29], so operations such as bulge, lean, shrink, and sag should also be represented in an initial vocabulary set.

To summarize, we list and define these operations for designers to begin to craft their unmaking as follows:

- $C R A C K$ - to break without complete separation

- SPLIT - to completely separate into distinct pieces

- $P I T$ - to undergo the formation of small pits on exterior surfaces, creating a patina of corrosion or textured indentations

- SHED - to undergo the removal or molting of a surface layer or outer covering

- DISSOLVE - to separate into atomic component parts and disintegrate

- BULGE - to swell or bend outward from exterior surfaces

- $L E A N$ - to bend in a particular direction (typically vertically)

- SHRINK - to decrease or contract in size or volume

- $S A G$ - to sink or bend downward from weight or pressure

This vocabulary is illustrated in Figure 2. This is only a starting set of essential unmaking operations; it is by no means a complete vocabulary. With some unmaking operations now defined, we next detail the technical parameters of various unmaking processes, demonstrate the vocabulary within CAD modeling software, and present working examples of actual resultant unmaking.

\section{3D PRINTING STRATEGIES FOR UNMAKING}

In this section, we provide a selection of 5 strategies that combine materials and techniques compatible with $3 \mathrm{D}$ printing to operationalize selected elements of the unmaking vocabulary. While 3D 
printing is not the only approach to exploring unmaking, the abilities to engineer hidden structures, deposit multiple materials on very small spatial scales, and generally execute designs with precision difficult to do by hand make it a valuable technique to create intriguing, sometimes unexpected manifestations of unmaking. We present our strategies in the context of "material selection strategies" - encompassing the single-material printing strategy and 2 types of multi-material printing strategies (overt and obscured) - and "structural design strategies" - encompassing the counter-stable mechanical design strategy and the triggered reactor design strategy. These strategies may be used on their own, but the most powerful demonstrations of unmaking will likely result when they, along with other future strategies, are used in concert with one another.

\subsection{Material Selection Strategies}

One of the most intuitive ways to engineer unmaking with any digital fabrication technique is to simply use materials that naturally exhibit desired unmaking operations. Broadly speaking, 3D printing strategies can either rely on printing with a single material or printing with multiple materials. Following convention, we define a "single material" to be a single input filament (for techniques such as fused deposition modeling), resin (for techniques such as sintered laser annealing), or powder (for techniques such as powder bed fusion) to create artifacts that appear monolithic in composition on a macroscopic scale; these input media themselves may be a single chemical compound or element, or they may comprise homogeneous blends of multiple compounds on a microscopic scale. "Multi-material" printing can be achieved with 3D printers outfitted with multiple or exchangeable extruders or write-heads, allowing for different parts of an object to be made from different input materials. The single-material printing and multi-material printing design strategies for unmaking are discussed subsequently.

5.1.1 Single-Material Printing. The single-material printing strategy uses a conventional single-material 3D printing approach with an input medium that is readily destructable or degradable. 3D printing objects with PVA, a common support material that dissolves in water, is an example of this approach (Figure 3(a)). Some other promising material candidates are biological materials, including food [28] and hydrogels comprising chitin, pectin, or other structural organic matter [29]. With powder bed fusion, a newer printing technique, corrosion-susceptible materials such as metals are also now 3D printable [40]. Finally, some "non-optimized" plastics - that is, those without the conventional additives that improve their mechanical properties - are prone to discoloration and embrittlement when exposed to UV light, water, or certain solvents and could also be leveraged to achieve more types oftextitcracking, pitting, or shedding effects.

This basic materials-centric approach can indeed produce some interesting unmaking effects. However, considering material choice alone is limiting, especially presently - that is, before the development of new materials that are optimized for unmaking. The unmaking vocabulary achievable by this approach is broad in theory but limited in practice. Identifying materials that are both compatible with digital fabrication flows and also easily degradable is currently challenging; most commercially available printable materials today are manufactured to resist unmaking, so unmaking using a single-material strategy can be difficult to induce, slow, or uncontrollable.

5.1.2 Multi-Material Printing (Overt). Multi-material printing strategies in general rely on the use of multiple input materials (filaments, resins, powders) to design artifacts that may non-uniformly degrade upon exposure to different catalysts, creating more complex effects than can be achieved with a single material. The overt multi-material printing strategy in particular refers to the approach of printing different parts of an artifact with different materials such that the differences in external colors and textures, while perhaps subtle, are perceptible; a close examination of the external surfaces of the initial object overtly reveals the multi-material nature of the object. With this strategy, one part may rust and shed away while another cracks and splits into pieces upon freezing. Figure 3(c) is one demonstration of this concept; iron-filled metal composite PLA filament (Proto-Pasta) is used to print one section of an object that selectively rusts upon exposure to salt water, while the plain PLA sections of the object remain unchanged. A multi-material approach affords a designer the ability to choreograph a multi-step, multi-catalyst unmaking process. An object might physically split when an intermediate part fails first, or it may become a patchwork of colors and pitted textures as it is subject to various elements. As is the case with single-material printing strategies, however, the richness of vocabulary achievable with this strategy is heavily dependent on the fundamental unmaking capabilities of the materials available.

5.1.3 Multi-Material Printing (Obscured). One of the unique capabilities of 3D printing is the ability to create internal structures that are not obvious upon external examination. The obscured multimaterial printing strategy is one in which an artifact appears to be printed from a single input material, but there are obscured internal chambers that are filled with a different "active material" - one that changes phase, shape, or otherwise releases energy upon exposure to a catalyst - to fracture or destruct those areas selectively. The entire object may also be a shell encapsulating a single chamber containing an active material that reacts with and transforms the shell material into an entirely new material with its own unmaking capabilities. A prototype inspired by this idea is shown in Figure 3(b); a model is printed with a wood-PLA blend filament (Timberfill PLA) shell and filled with a hemp-mycelium spore blend (which can be printed as a paste [22]); with proper humidity and oxygen, the mycelium colonizes the shell material, changing its appearance and mechanical properties.

We explore another manifestation of the obscured multi-material design strategy in more detail in upcoming sections of this paper. This strategy is especially useful in creating surprising and delightful unmaking experiences post-making. By modulating the geometry of internal chambers and the selection of the active material, we can design for a swath of unmaking operations, including splitting, bulging, and leaning. These capabilities can even be rendered such that the maker of such artifacts can be unaware of the hidden unmaking that is embedded.

\subsection{Structural Design Strategies}

In addition to the careful selection of materials used for printing, structural design strategies are also key to enabling our unmaking 

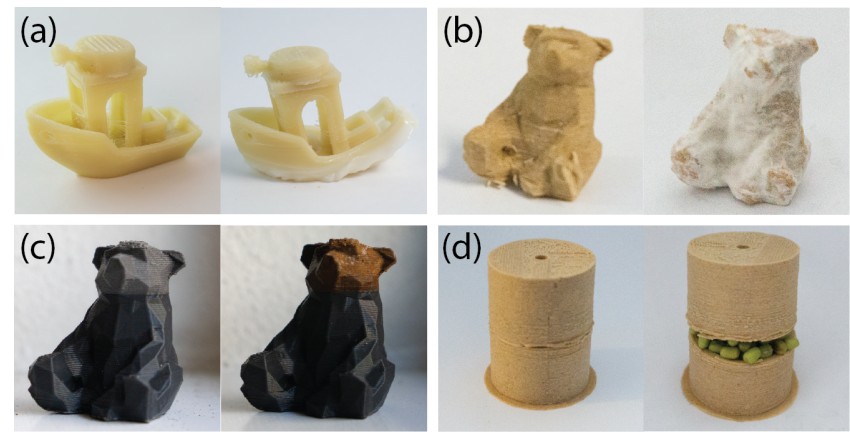

Figure 3: Demonstrations of different unmaking strategies. (a) Single material printing: A PVA model is selectively dipped in water; water is absorbed and causes melting and splitting. (b) Multi-material printing (obscured): A Timberfill wood-PLA composite shell is filled with mycelium spores; mycelium colonizes the shell material, softening it and drastically changing its appearance. (c) Multi-material model printing (overt): A model comprising solid PLA for the body and iron-PLA composite for the head; when exposed to salt water, the head selectively rusts. (d) Obscured multi-material printing + counter-stable mechanical design: A wood-PLA composite shell is filled with hydrated mung beans; the sprouting of the mung beans causes cleavage along a pre-defined weak layer in the cylinder.

vocabulary. Possible strategies, discussed subsequently, include counter-stable mechanical design and triggered reactor design.

5.2.1 Counter-Stable Mechanical Design. Counter-stable mechanical design strategies abandon the rules of stable design from mechanical engineering and print with intentionally-created mechanical weak parts. For example, we can print layers that are especially thin or fragile, or adjust print parameters to intentionally create marginal designs. Figure 3(d) illustrates this approach in combination with an obscured multi-material printing strategy; a Timberfill wood-PLA composite hollow cylinder is printed using a singleextruder Fused Deposition Modeling (FDM) 3D printer, and a weak layer is created by skipping a print layer in the printer's machine code. Sprouting mung beans placed inside the cylinder cause the cylinder to crack along the weak layer after a few days. Counterstable mechanical design may be made overt to varying degrees. Weaknesses may be obvious to virtually anyone through the creation of external gaps in material, thin connections, or wrongly moving parts, perhaps even suggesting and inviting a particular interaction to catalyze unmaking. Alternatively, they may require the trained eye of a structural or mechanical engineer. They may also be completely obscured using, for instance, the creation of hidden, internal volumes that are unsupported, setting the stage for unmaking that may occur by random, unintentional interactions.

Using a counter-stable mechanical design strategy, and generally rethinking printing strategies in addition to material selection, can allow a design to transcend perceived material limitations and be used to simulate unexpected unmaking operations. 3D printing technologies enable us to engineer an object with sub-millimeter, layer-by-layer precision, so there is no reason to simply wait for the "right" materials to be developed before exploring unmaking. Plastics, a workhorse of 3D printing, are inherently soft materials that exhibits ductile failure, characterized by deformation before breakage. In addition to using materials-based strategies to enable unmaking in this this way (sagging or leaning), the counter-stable mechanical design strategy allows us to instead emulate brittle fracture - characterized by cracking and splitting along clean cleavage surfaces (such as the weak layer in Figure 3(d)) without noticeable deformation - that is normally only expected from non-plastic materials such as metals, ceramics, and glass.

5.2.2 Triggered Reactor Design. The triggered reactor design strategy uses a combination of chemical catalysts and mechanical design to engineer a chemical reactor that triggers unmaking upon being manipulated in a specified way or after a certain amount of time. By nature, this strategy as described is also a multi-material one. For example, baking soda and vinegar may be deposited into separate chambers within a 3D printed object and be forced to later combine, either when turned upside down by a human or when an internal dividing structure dissolves after a set amount of time. Once combined, the chemicals react to form carbon dioxide gas that builds up pressure and, after a few seconds, suddenly bursts through part of the object. A video of this example is in our Supplemental Materials. By engineering how internal structures are designed, unmaking may be triggered by other specific manipulations, such as spinning, being dropped, or being squeezed. By tweaking the concentrations of chemicals used and type of reaction induced, the duration of unmaking can also be modulated from milliseconds to years. This strategy might be used to realize unmaking vocabulary such as splitting, bulging, shrinking, and dissolving.

\section{OPERATIONALIZING UNMAKING}

We operationalize the concept and design vocabulary of unmaking by demonstrating its creative process, resulting artifact, and the eventual unmaking experience using a multi-material 3D printing process. We use an obscured multi-material design strategy to print chambers of an active material within a primary material, demonstrating that by varying the geometry and placement of the chambers and conditions of unmaking, we can induce different, controllable unmaking effects, exemplifying splitting and bulging in particular. Controllability is an important characteristic of unmaking, as we are interested not in simply "blowing things up" but rather in how designers can "build in" desired unmaking parameters into their designs. Of course, we want to embrace elements of chance and entropy as well and allow for some variation in the timing and precise geometry of unmaking. We present our system not as an ideal example that embodies all of the aforementioned potential of unmaking design but rather as one that, despite being limited by current material sets, provides a preview into what can be achieved.

\subsection{Materials}

For our demonstration, we use off-the-shelf materials to provide a glimpse into what is already possiblefrom an artistic perspec- 

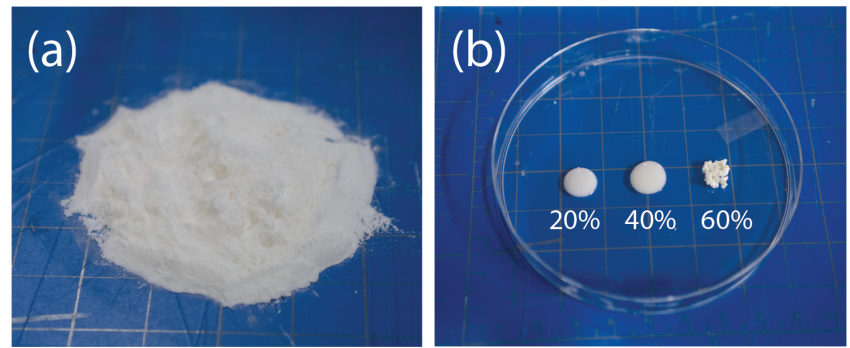

Figure 4: Images of (a) dry microsphere powder and (b) prepared 20, 40, and $60 \mathrm{wt} \%$ water-based slurries

tive, even before the development of materials particularly designed to support "unmaking." We fabricate PLA objects with selectively placed chambers filled with our active material of choice thermally-expanding microspheres. Thermally-expanding microspheres are composed of thermoplastic shells encapsulating a low boiling point hydrocarbon. Upon heating to a temperature within an activation window, the hydrocarbon expands while the thermoplastic shell softens, causing the microspheres to swell like balloons to up to 60 times their original volume. These microspheres are conventionally used as blowing agents in automotives, construction, packaging, and coatings to reduce weight and density. In research, they have also been used in ExpandFab, a 4D-printing method for fabricating expanding foam artifacts [20], and in transient electronics explorations for triggering the shattering of microchips [2]. The microspheres used for our demonstrations (Nouryon Expancel 043 DU 80) are of proprietary composition and assumed to be hazardous, but Nouryon, the provider of our microspheres and one of the world's largest manufacturers of expanding microspheres, announced in 2019 that they had developed prototypes of microspheres made from cellulose - a bio-based, readily-degradable compound that could serve the same purpose as the microspheres reported here [41].

The microspheres we use here have a diameter $16-24 \mu \mathrm{m}$, a starting activation temperature of $95-115^{\circ} \mathrm{C}$, and a maximum temperature of $147-167^{\circ} \mathrm{C}$. Different varieties of microspheres with different size and temperature ranges can be used to achieve the effects we demonstrate subsequently, but their activation temperature should not exceed the melting temperature of the main printing filament to avoid uncontrolled warpage and melting.

We prepare a slurry of microspheres by mixing them in water to make them compatible with standard paste-based extrusion methods. We print with a concentration of $40 \mathrm{wt} \%$. Higher concentrations make the microsphere slurry difficult to print and result in an excess mass of expanded microspheres upon destruction. Lower concentrations may result in unintentionally incomplete unmaking effects, the obvious seepage of loose slurry from 3D prints before and during unmaking, and uneven effects over time due to a decrease in slurry volume as water evaporates. Images of the dry microspheres and prepared slurries of various concentrations are shown in Figure 4. Microspheres slurries of concentrations greater than $40 \mathrm{wt} \%$ maintain their volume over time when left undisturbed, even after the water has evaporated.
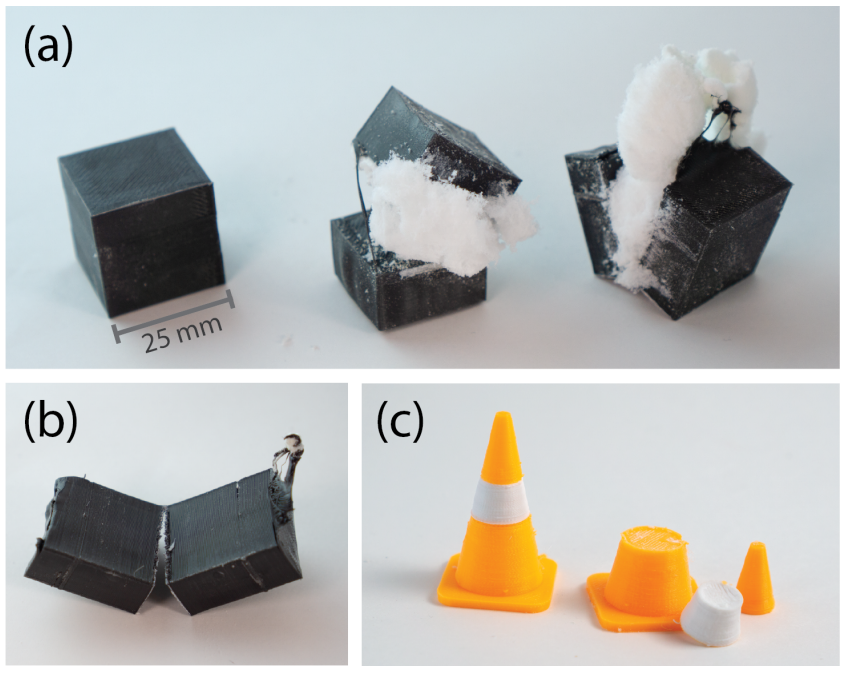

Figure 5: (a) PLA cubes can be split parallel to print layers OR perpendicular to print layers. (b) The surfaces of the split PLA pieces after microspheres are brushed off are smooth. (c) Splitting may be used to separate multi-color (or multimaterial) artifacts cleanly into their constituent parts for recycling.

\subsection{Splitting}

One of the most important operations of unmaking that is realizable by our approach is splitting. Figure 5 shows the results of defining and printing a thin plane of microspheres inside FDM-printed PLA models. The plane of microspheres is $1 \mathrm{~mm}$ thick and extends nearly to the object's external surfaces, with an offset margin of $0.5 \mathrm{~mm}$. After printing, the objects with various internal splitting planes appear identical. However, upon heating to $130^{\circ} \mathrm{C}$ for 10 minutes, the objects split along the defined planes. While FDMprinted objects by nature are more susceptible to breakage along print line boundaries, Figure 5 shows that objects may be broken against print lines as well with our method. The resulting expanded microspheres may be left as-is or cleaned off with water or a dry brush.

Practically, this method may be used to divide parts into their constituent materials or colors (Figure 5(c)), which is important to maintain renewed material quality upon recycling and reuse; layers of microspheres may be inserted at each material boundary to accomplish this. If this is the primary goal, a designer may wish to explicitly prescribe unmaking to the makers and "users" of an artifact, as the artifact itself carries no signifiers of the affordance of unmaking. On the other hand, this method can also be used to intentionally create more mysterious, startling unmaking effects. The "obscured" aspect of this obscured multi-material design strategy - the fact that the unmaker has no indication of where and how the object will break until the moment it happens - contributes to the suspension, surprise, and perhaps delight that is evoked when the object is placed in an oven or on a hotplate and splits unexpectedly. In fact, because the unmaking that the designer has embedded is invisible, the object may unmake days, months, or 

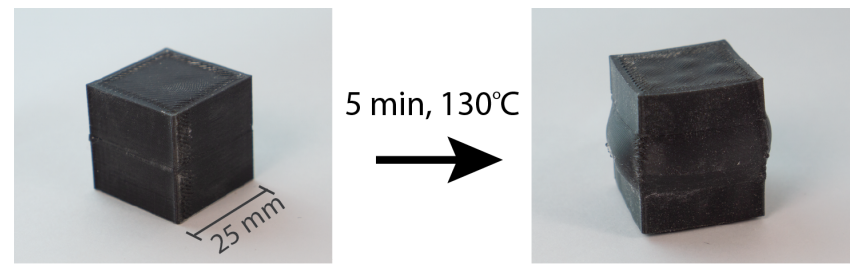

Figure 6: A pre-defined section of a PLA cube bulges upon heating.

years later. Conceptually, this is an important new design parameter - the fact that a design is not "done" simply once it is made. The intended design is only fully realized when the object's unmaking is manifested. The tension, anxiety, and delight embodied in this experience are a central characteristic of unmaking.

Additionally, the splitting of PLA objects appears to violate expected modes of mechanical failure that should result from "immutable" material properties. Although textbooks and material properties tables indicate that PLA breakage resulting from heat or mechanical stress occurs via ductile failure, as seen in Figure 5, the end result of our splitting operation instead resembles brittle fracture, with slight deformation visible only on the edges of the cleavage surfaces.

\subsection{Bulging}

It may also be desirable to celebrate unmaking in an intermediate state prior to an object's separation into pieces. Much of our unmaking vocabulary defining various forms of deformation captures such states; here, we discuss the realization of bulging. With our approach, we can set the stage for highly anticipatory future unmaking experiences by inducing bulging in an initial unmaking experience. Like splitting, bulging can be surprising and unexpected due to the lack of outward-facing material or structural weaknesses. Upon witnessing the object bulge, an individual may halt unmaking and decide to interact with or display the deformed object for some time. Once an object has bulged, or more generally deformed (e.g. sagged, leaned, or shrunken), its potential for unmaking is no longer obscured. While the initial unmaking session may have evoked shock or intrigue, the subsequent session may be one of anticipation and satisfaction.

To implement bulging, we define and fill larger chambers of microspheres. Figure 6 shows the results of defining and printing a large $(24 \times 24 \times 5 \mathrm{~mm}$ inside a $25 \times 25 \times 25 \mathrm{~mm}$ cube) chamber of microspheres (40 wt \% slurry). The cube was heated at $130^{\circ} \mathrm{C}$ for 5 minutes.

In addition to heating time, microsphere slurry concentration and heating temperature can modulate the speed and amount of bulging that happens. Figure 7 shows hollow PLA test pieces $(15 \times 15 \times 10$ $\mathrm{mm}$ with a wall thickness of $0.5 \mathrm{~mm}$ ) (a) filled with slurries of microsphere concentrations ranging from $20 \%$ to $60 \%$ by weight and heated at $130^{\circ} \mathrm{C}$ for 5 minutes; and (b) filled with a $40 \mathrm{wt} \%$ microsphere slurry and heated to temperatures ranging from $110^{\circ} \mathrm{C}$ to $150^{\circ} \mathrm{C}$ for 5 minutes. The amount of bulging increases with heating time, heating temperature, and the concentration of microspheres

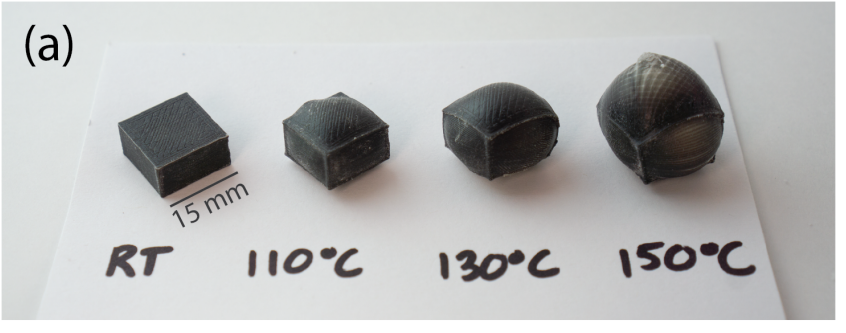

(b)

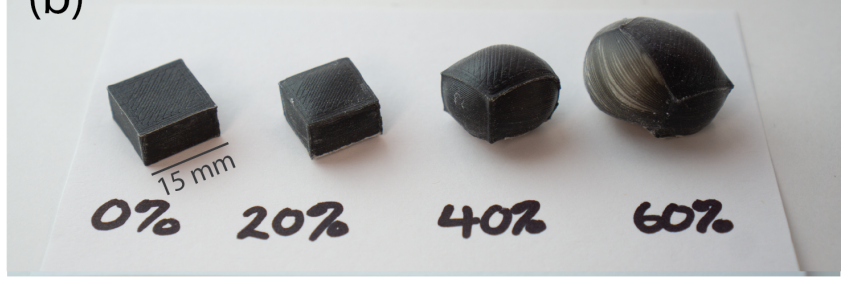

Figure 7: The degree of bulging may be modulated with (a) heating temperature (fixing microsphere slurry concentration at $40 \mathrm{wt} \%$ and heating time at 5 minutes) or (b) microsphere slurry concentration (fixing heating temperature at $130^{\circ} \mathrm{C}$ and heating time at 5 minutes).

used. The designer and the owner of an object (when they are different people) can thus collaboratively shape the unmaking of their objects.

\section{3D MODELING DESIGN TOOL}

Carrying out unmaking design strategies in practice can be laborious and nearly impossible if every artifact is modeled from scratch. To overcome this and support widespread unmaking design exploration, we need to develop software tools that operationalize the unmaking vocabulary. Such tools should also require as little extra input from the designer as possible to execute the details of generating printable meshes, abstracting out the mechanical and chemical know-how required to implement the designer's desired unmaking effect(s). On a high level, this involves developing an abstracted module for each unmaking operation. A designer can then can simply select desired unmaking operations from a menu to drop into their 3D models. In this section, we present example unmaking CAD macros for our PLA and microspheres system. However, this tool is not material-dependent and can be used for unmaking designs with other material combinations that use the obscured multi-material design recipe as well.

Users can design the unmaking of artifacts in the 3D-modeling program Rhinoceros (Rhino) using our custom macros. In the example shown in Figure 8, we demonstrate splitting, the unmaking operation in which an object cleaves along a specified surface. We have also made a macro for bulging, the effect in which an object swells in a given area but does not physically break. The parametric macros are scripted in Rhino.Python. ${ }^{1}$ To illustrate this, we walk through the usage of the splitting macro. The designer creates or loads their mesh model in Rhino and defines a surface (or surfaces)

\footnotetext{
${ }^{1}$ https://github.com/kwsong/unmaking_public
} 


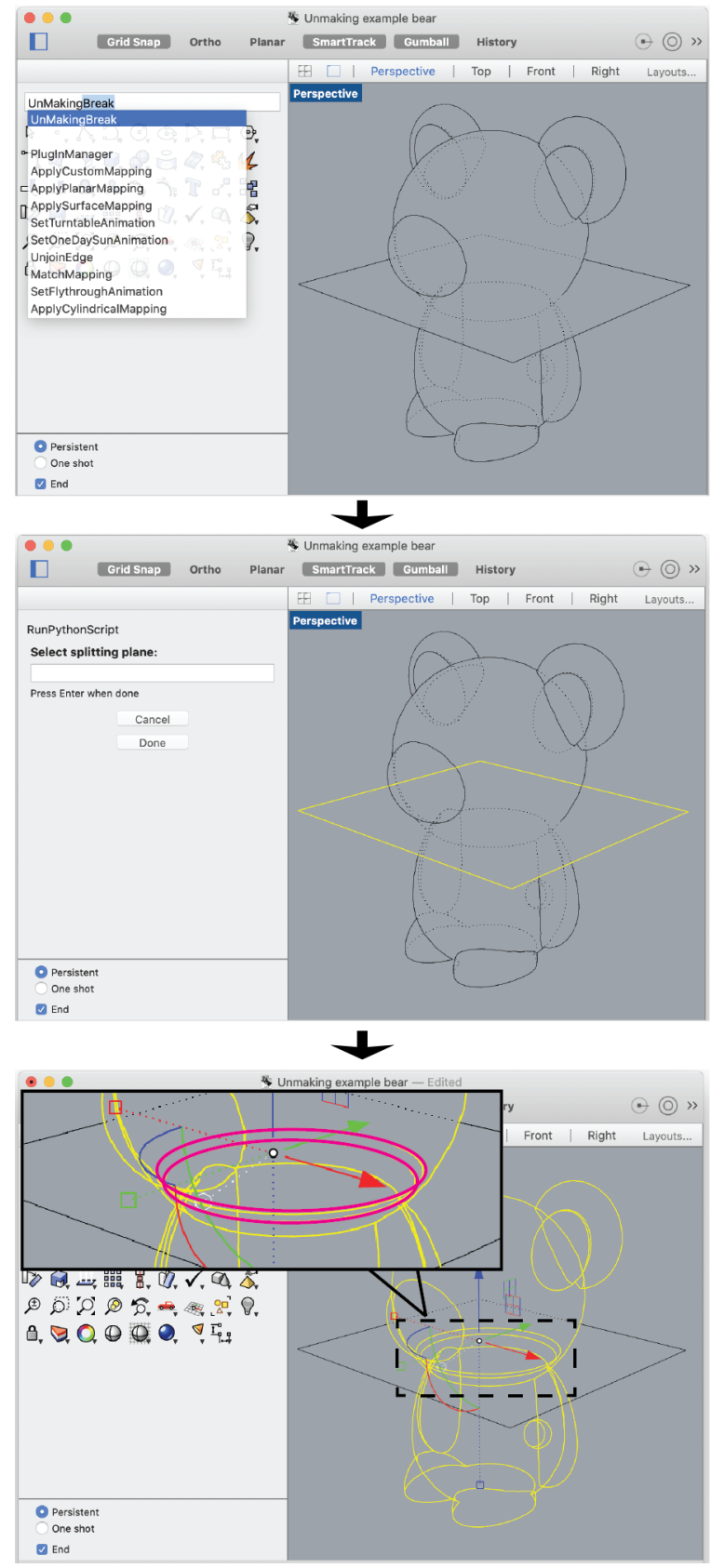

Figure 8: A custom Rhino macro allows the designer to define surfaces along which a 3D model should break and generates modified meshes that can be exported as .stl models to be sliced, 3D printed, and unmade. An inset highlights the added mesh boundaries in pink.

that the model should cleave along when heated. The surfaces do not have to be planar. The macro prompts the user to select these elements and automatically generates 2 printable meshes with the correct face normals: one that is the original model with a hollowed out chamber centered on the user-defined splitting surface (the

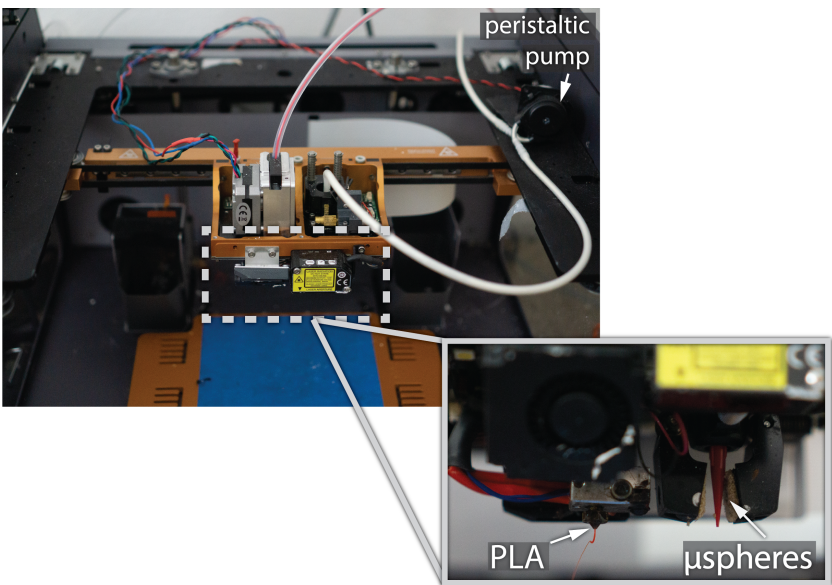

Figure 9: 3D printer setup with PLA printed via FDM and microspheres extruded with a peristaltic pump

chamber is $1 \mathrm{~mm}$ thick and $0.5 \mathrm{~mm}$ offset from the external surface of the model), and one that is the chamber itself (defining the microsphere volume). The resulting 3D models can then be exported as .stl files from Rhino to Slic3r, an open-source slicing program that converts the model into machine gcode to be interpreted by the 3D printer. The bulging macro operates in a likewise fashion, with the addition of an intermediate dialog in which the user specifies the thickness of the bulging chamber after selecting the base surface.

Such an idea can be expanded to support a greater range of unmaking operations, as well as different unmaking design strategies. For example, for obscured multi-material designs, we might also induce leaning by generating asymmetrical chambers based on a desired leaning arc that the designer draws. For counter-functional mechanical designs, we might take as an input a user-defined volume and convert solid parts of a model lying inside that volume to a fragile wireframe. For triggered reactor designs, we might use a user-defined volume to generate appropriately sized and placed U-shaped chambers, to be filled with chemicals that mix when the chambers are inverted. These designs can be chained together to create wildly creative and unexpected unmaking effects.

\section{FABRICATION PROCESS}

A wide variety of 3D printing technologies, including fused deposition modeling (FDM), selective laser sintering (SLS), stereolithography (SLA), and PolyJet 3D printing, support unmaking. In our examples, we use a dual extruder 3D printer (modified Voxel8), with the primary extruder printing thermoplastic filament via a standard FDM process and the secondary extruder outfitted with a syringe whose flow is controlled by a peristaltic pump (Figure 9). PLA is printed on the primary extruder at $200^{\circ} \mathrm{C}$, and the microsphere slurry is printed on the secondary extruder at room temperature.

Some of the prototypes shown in this paper were printed on single-material 3D printers, and for these, we paused printing to hand-pipe the microsphere slurry into chamber(s) using a syringe for convenience. Virtually any deposition or extrusion-based 3D printer can be easily modified to print microsphere slurries with commercially available components and systems [7, 61]. 


\section{DISCUSSION}

The method that we have presented is an example of an obscured multi-material design strategy relying on readily available off-theshelf materials that enables surprising, and perhaps even shocking, unmaking experiences post-making. We have shown that we can realize a subset of our unmaking vocabulary, including splitting and bulging, and are confident that future efforts will realize other operations as well. Still, it is clear that the design potential of unmaking can be most fully realized with materials specially developed with unmaking in mind. Unmaking with commercially-available thermally-expanding microspheres has its limitations. Because the activation temperature of our microspheres is relatively high, unmaking with this method requires human input; microspheres with activation temperatures as low as $80^{\circ} \mathrm{C}$ exist, but $80^{\circ} \mathrm{C}$ still exceeds most "in-the-wild" conditions.

Additionally, with regards to sustainability considerations, while our method as-is can be helpful for separating different colored PLA sections and potentially breaking PLA artifacts into small pieces for easier and higher-quality recycling, the claim of our method as "sustainable" admittedly cannot be truly realized until we replace the microspheres with Nouryon's hopefully imminent cellulose (or equivalent) microspheres [41]. We also must replace PLA with a material that is degradable with consumer unmaking techniques alone so that we do not rely on energy-intensive industrial recycling or composting processes. Arguably, such easily biodegradable materials are not commercially available today due to lack of compelling demand. While initially discouraging, we believe that a growing interest in unmaking could accelerate the development of these materials and compatible processes. One promising example of a material and technique under active investigation is Gladman et al.'s 3D printing of cellulose-based hydrogel structures with a direct-ink writing technique [46]; such structures might be seeded or filled with enzymes or bacteria that could induce selective unmaking without hazardous or excess byproducts. As previously described, we envision unmaking as a collaborative process between a designer and owner of an object that continuously creates new, personalized value in an object as it destructs and returns back to the earth, reducing the reliance on energy-intensive industrial recycling processes and potentially eliminating the concept of disposal altogether. We hope that our presentation of unmaking as an under-explored design space will increase interest in the usage of and incentivize more research and commercial development of easily degradable materials.

Still, even as presented, the unmaking concept enables and perhaps even forces designers to think about and consider how their objects should fail and destruct - something currently not often considered. Our demonstrations only scratch the surface of what is possible with not only future materials but also other digital fabrication techniques, including ones that are subtractive instead of additive processes. Laser cutting and CNC milling are popular digital fabrication technologies that use a laser beam or mechanical cutter to selectively etch away parts of a starting mass of material. While obscured multi-material strategies are perhaps not easily adapted to such processes, the single-material and counter-stable mechanical design strategies can be readily deployed. By selecting a readily degradable material or by carving the initial material out to create mechanically weak areas, subtractive fabrication technologies can be used for unmaking in a similar way for these strategies that we have already described. We believe that designers will continue to explore and find other novel strategies for unmaking that uniquely lend themselves to particular digital fabrication technologies as well.

Next, we present 3 theoretical scenarios to illustrate what the maturation of unmaking materials and strategies could enable.

\subsection{Scenario 1: Enabling Evolving Design}

A tomato plant has outgrown its small 3D-printed starter pot and is repotted, leaving the starter pot empty and unused. Fortunately, the pot was designed for unmaking. UV exposure from weeks of sitting outside has caused the pot to be broken into a collection of items, such as a saucer for the larger pot and stakes for the growing plant. As the tomato plant continues to grow and render these pieces useless, the pieces can be microwaved and broken into smaller but still usable parts. This process of creating new forms through unmaking can continue until the pieces are small enough such that they mix into and enrich the soil of the tomato plant.

\subsection{Scenario 2: Celebrating Sustainability}

A large, transparent drum teeming with a colorful potpourri of objects sits at the front of a community makerspace where the dull, black trash bins used to greet makers. Unmakers can feed their used prototypes, failed prints, partially unmade objects, or scraps into this drum. Passerbys can stop by to simply admire the colorful medley of items currently in the drum, add water to the drum and watch in delight as some objects melt away while others seemingly appear out of nowhere in front of them, heat the drum and watch other objects expand and pop, or spin the drum to observe other objects buckle and snap. Colorful bacterial colonies in the drum slowly feed on yet other objects, reducing some to pieces and giving birth to new life forms. Eventually, every object becomes compost material that is sifted into a separate tray for anyone to collect for their garden. The concept of a trash bin has transformed into one of an interactive, tangible, and living display of materials transitioning from their use to end-of-life phases.

\subsection{Scenario 3: Creating Aura}

A 3D-printed clock made decades ago by an unmaking designer in the family hangs proudly in the living room. Once a generic, circular piece placed on a desk simply to tell time, its appearance now embodies family secrets and stories. Like out of a Salvador Dalí painting, the numbers of the clock have partially melted and dripped away from the unusually hot summer 15 years ago. Orange swirls of rust adorn the face of the clock and become more vibrant and etched with each passing year. From years of jostling around in moving boxes, being misused as a frisbee, and falling off the wall, faint cracks have formed. The family has a favorite story about certain fractures that had suddenly - accompanied, as the story goes, with dramatic crackling and whistling - become accentuated a few years ago when the clock had been accidentally hung sideways for a week. These are particularly intriguing; they seem to be revealing some kind of message from a bygone generation, but one cannot be sure...not yet, anyway. The clock has long ago ceased to be able 
to tell time, but it has nonetheless become a one-of-a-kind family heirloom for what it represents and evokes and what it may reveal in the future.

\section{CONCLUSION}

This paper presents unmaking - the destruction, decay, and deformation - of physical artifacts as a valuable extension to making. Artists and HCI researchers alike have long heralded the personal values and meanings that destruction as an aesthetic and act can bestow upon objects. Still, typically, great effort goes into ensuring that designs endure and avoid destruction. We respond to this discourse by offering an unmaking vocabulary - crack, split, pit, shed, dissolve, bulge, lean, shrink, and sag - for digital fabrication technologies, particularly 3D printing, to operationalize the controlled unmaking of physical artifacts. Combining material selection and structural design, we outline 5 design strategies for the unmaker: single-material design, overt multi-material design, obscured multi-material design, counter-stable mechanical design, and triggered reactor design. We present an obscured multi-material design strategy that places expanding microspheres inside PLA structures, developing an accompanying software tool to modify 3D models and demonstrating that our strategy operationalizes unexpected unmaking operations, including splitting and bulging.

Future work in unmaking includes the fulfillment of a wider array of our unmaking vocabulary through both software design tools and material explorations. Additionally, we also have an environmental obligation to focus future work particularly on realizing the potential of unmaking design to foster sustainable practices and material development. We hope to build upon and contribute to the development of biological materials [21, 29, 46, 52, 53, 58], focusing on incorporating them into mainstream digital fabrication workflows so that their unique unmaking aesthetics may be realized and used to inspire the development of new materials.

\section{ACKNOWLEDGMENTS}

We are grateful for the generous support from the NDSEG Fellowship program and the UC Berkeley Jacobs Institute Innovation Catalysts initiative. We also acknowledge the Nouryon and Kureha Corporations for generously supplying expanding microspheres. Finally, we thank the thoughtful reviewers for their constructive feedback on this research.

\section{REFERENCES}

[1] Byoungkwon An, Hsiang-Yun Wu, Teng Zhang, Lining Yao, Ye Tao, Jianzhe Gu, Tingyu Cheng, Xiang 'Anthony' Chen, Xiaoxiao Zhang, Wei Zhao, Youngwook Do, and Shigeo Takahashi. 2018. Thermorph: Democratizing 4D Printing of Self-Folding Materials and Interfaces. In Proceedings of the 2018 CHI Conference on Human Factors in Computing Systems - CHI '18. ACM Press, Montreal QC, Canada, 1-12. https://doi.org/10.1145/3173574.3173834

[2] N. Banerjee, Y. Xie, Md. M. Rahman, H. Kim, and C. H. Mastrangelo. 2014. From chips to dust: The MEMS shatter secure chip. In 2014 IEEE 27th International Conference on Micro Electro Mechanical Systems (MEMS). IEEE, San Francisco, CA USA, 1123-1126. https://doi.org/10.1109/MEMSYS.2014.6765843

[3] Walter Benjamin. 1935. The Work of Art in the Age of Mechanical Reproduction, 1936.

[4] Eli Blevis. 2007. Sustainable interaction design: invention \& disposal, renewal \& reuse. In Proceedings of the SIGCHI Conference on Human Factors in Computing Systems - CHI '07. ACM Press, San Jose, California, USA, 503-512. https://doi. org $/ 10.1145 / 1240624.1240705$

[5] J. William Boley, Wim M. van Rees, Charles Lissandrello, Mark N. Horenstein, Ryan L. Truby, Arda Kotikian, Jennifer A. Lewis, and L. Mahadevan. 2019. Shapeshifting structured lattices via multimaterial $4 \mathrm{D}$ printing. Proceedings of the
National Academy of Sciences 116, 42 (Oct. 2019), 20856-20862. https://doi.org/ 10.1073/pnas.1908806116

[6] Hronn Brynjarsdottir, Maria Håkansson, James Pierce, Eric Baumer, Carl DiSalvo, and Phoebe Sengers. 2012. Sustainably unpersuaded: how persuasion narrows our vision of sustainability. In Proceedings of the 2012 ACM annual conference on Human Factors in Computing Systems - CHI '12. ACM Press, Austin, Texas, USA, 947. https://doi.org/10.1145/2207676.2208539

[7] byFlow. 2020. 3D Food Printer. https://www.3dbyflow.com. Accessed: 2020-09-10.

[8] Marina F. Cosate de Andrade, Patrícia M. S. Souza, Otávio Cavalett, and Ana R. Morales. 2016. Life Cycle Assessment of Poly(Lactic Acid) (PLA): Comparison Between Chemical Recycling, Mechanical Recycling and Composting. Fournal of Polymers and the Environment 24, 4 (Dec. 2016), 372-384. https://doi.org/10. 1007/s10924-016-0787-2

[9] Haitao Cui, Shida Miao, Timothy Esworthy, Se-jun Lee, Xuan Zhou, Sung Yun Hann, Thomas J. Webster, Brent T. Harris, and Lijie Grace Zhang. 2019. A novel near-infrared light responsive $4 \mathrm{D}$ printed nanoarchitecture with dynamically and remotely controllable transformation. Nano Research 12, 6 (June 2019), 1381-1388. https://doi.org/10.1007/s12274-019-2340-9

[10] Emily C. Davidson, Arda Kotikian, Shucong Li, Joanna Aizenberg, and Jennifer A. Lewis. 2020. 3D Printable and Reconfigurable Liquid Crystal Elastomers with Light-Induced Shape Memory via Dynamic Bond Exchange. Advanced Materials 32, 1 (Jan. 2020), 1905682. https://doi.org/10.1002/adma.201905682

[11] Laura Devendorf and Daniela K. Rosner. 2015. Reimagining Digital Fabrication as Performance Art. In Proceedings of the 33rd Annual ACM Conference Extended Abstracts on Human Factors in Computing Systems - CHI EA '15. ACM Press, Seoul, Republic of Korea, 555-566. https://doi.org/10.1145/2702613.2732507

[12] Kristin N. Dew, Samantha Shorey, and Daniela Rosner. 2018. Making within limits: towards salvage fabrication. In Proceedings of the 2018 Workshop on Computing within Limits - LIMITS '18. ACM Press, Toronto, Ontario, Canada, 1-11. https: //doi.org/10.1145/3232617.3232626

[13] Carl DiSalvo, Phoebe Sengers, and Hrönn Brynjarsdóttir. 2010. Mapping the landscape of sustainable HCI. In Proceedings of the 28th international conference on Human factors in computing systems - CHI '10. ACM Press, Atlanta, Georgia, USA, 1975. https://doi.org/10.1145/1753326.1753625

[14] Anthony A. D'Amico, Analise Debaie, and Amy M. Peterson. 2017. Effect of layer thickness on irreversible thermal expansion and interlayer strength in fused deposition modeling. Rapid Prototyping fournal 23, 5 (Aug. 2017), 943-953. https://doi.org/10.1108/RPJ-05-2016-0077

[15] David Eickhoff, Stefanie Mueller, and Patrick Baudisch. 2016. Destructive Games: Creating Value by Destroying Valuable Physical Objects. In Proceedings of the 2016 CHI Conference on Human Factors in Computing Systems - CHI '16. ACM Press, Santa Clara, California, USA, 3970-3974. https://doi.org/10.1145/2858036. 2858113

[16] Elisa Giaccardi, Elvin Karana, Holly Robbins, and Patrizia D’Olivo. 2014. Growing traces on objects of daily use: a product design perspective for HCI. In Proceedings of the 2014 conference on Designing interactive systems - DIS '14. ACM Press, Vancouver, BC, Canada, 473-482. https://doi.org/10.1145/2598510.2602964

[17] Bona Goo, Chae-Hui Hong, and Keun Park. 2020. 4D printing using anisotropic thermal deformation of 3D-printed thermoplastic parts. Materials \& Design 188 (March 2020), 108485. https://doi.org/10.1016/j.matdes.2020.108485

[18] Jianzhe Gu, David E. Breen, Jenny Hu, Lifeng Zhu, Ye Tao, Tyson Van de Zande, Guanyun Wang, Yongjie Jessica Zhang, and Lining Yao. 2019. Geodesy: Self-rising 2.5D Tiles by Printing along 2D Geodesic Closed Path. In Proceedings of the 2019 CHI Conference on Human Factors in Computing Systems - CHI '19. ACM Press, Glasgow, Scotland Uk, 1-10. https://doi.org/10.1145/3290605.3300267

[19] Miwa Ikemiya and Daniela K. Rosner. 2014. Broken probes: toward the design of worn media. Personal and Ubiquitous Computing 18, 3 (March 2014), 671-683. https://doi.org/10.1007/s00779-013-0690-y

[20] Hiroki Kaimoto, Junichi Yamaoka, Satoshi Nakamaru, Yoshihiro Kawahara, and Yasuaki Kakehi. 2020. ExpandFab: Fabricating Objects Expanding and Changing Shape with Heat. In Proceedings of the Fourteenth International Conference on Tangible, Embedded, and Embodied Interaction. ACM, Sydney NSW Australia, 153-164. https://doi.org/10.1145/3374920.3374949

[21] Elvin Karana, Davine Blauwhoff, Erik-Jan Hultink, and Serena Camere. 2018. When the Material Grows: A Case Study on Designing (with) Mycelium-based Materials. International fournal of Design 12, 2 (2018), 18

[22] Eric Klarenbeek. 2011. The Mycelium Project - Print and Grow. = https://ericklarenbeek.com.

[23] Cindy Kohtala. 2017. Making "Making" Critical: How Sustainability is Constituted in Fab Lab Ideology. The Design fournal 20, 3 (May 2017), 375-394. https: //doi.org/10.1080/14606925.2016.1261504

[24] Leonard Koren. 2008. Wabi-sabi for artists, designers, poets \& philosophers. Imperfect Publishing, Point Reyes, CA, USA.

[25] Eldy S. Lazaro Vasquez, Hao-Chuan Wang, and Katia Vega. 2020. Introducing the Sustainable Prototyping Life Cycle for Digital Fabrication to Designers. In Proceedings of the 2020 ACM Designing Interactive Systems Conference. ACM, Eindhoven Netherlands, 1301-1312. https://doi.org/10.1145/3357236.3395510 
[26] Steven K. Leist, Dajing Gao, Richard Chiou, and Jack Zhou. 2017. Investigating the shape memory properties of $4 \mathrm{D}$ printed polylactic acid (PLA) and the concept of $4 \mathrm{D}$ printing onto nylon fabrics for the creation of smart textiles. Virtual and Physical Prototyping 12, 4 (Oct. 2017), 290-300. https://doi.org/10.1080/17452759. 2017.1341815

[27] Steven K. Leist and Jack Zhou. 2016. Current status of 4D printing technology and the potential of light-reactive smart materials as $4 \mathrm{D}$ printable materials. Virtual and Physical Prototyping 11, 4 (Oct. 2016), 249-262. https://doi.org/10. 1080/17452759.2016.1198630

[28] Ying-Ju Lin, Parinya Punpongsanon, Xin Wen, Daisuke Iwai, Kosuke Sato, Marianna Obrist, and Stefanie Mueller. 2020. FoodFab: Creating Food Perception Illusions Using Food 3D Printing. In Proceedings of the 2020 CHI Conference on Human Factors in Computing Systems (Honolulu, HI, USA) (CHI '20). Association for Computing Machinery, New York, NY, USA, 1-13. https: //doi.org/10.1145/3313831.3376421

[29] Andrea Ling. 2018. Design by Decay, Decay by Design. Master's thesis. MIT Media Lab.

[30] Szu-Yu (Cyn) Liu. 2017. To Decompose Is to Create: Supporting Creativity by Incorporating Nature in Design. In Proceedings of the 2017 ACM SIGCHI Conference on Creativity and Cognition. ACM, Singapore Singapore, 484-489. https://doi.org/10.1145/3059454.3078696

[31] Szu-Yu (Cyn) Liu, Jeffrey Bardzell, and Shaowen Bardzell. 2019. Decomposition as Design: Co-Creating (with) Natureculture. In Proceedings of the Thirteenth International Conference on Tangible, Embedded, and Embodied Interaction - TEI '19. ACM Press, Tempe, Arizona, USA, 605-614. https://doi.org/10.1145/3294109. 3295653

[32] Michal Luria, Ophir Sheriff, Marian Boo, Jodi Forlizzi, and Amit Zoran. 2020 Destruction, Catharsis, and Emotional Release in Human-Robot Interaction. ACM Transactions on Human-Robot Interaction 9, 4 (June 2020), 1-19. https://doi.org/ $10.1145 / 3385007$

[33] Jennifer C. Mankoff, Eli Blevis, Alan Borning, Batya Friedman, Susan R. Fussell, Jay Hasbrouck, Allison Woodruff, and Phoebe Sengers. 2007. Environmental sustainability and interaction. In CHI '07 extended abstracts on Human factors in computing systems - CHI '07. ACM Press, San Jose, CA, USA, 2121. https: //doi.org/10.1145/1240866.1240963

[34] Claire Martin. 2016. Anger Rooms: A Smashing New Way to Relieve Stress. The New York Times Section BU (26 Nov. 2016), 4. https://www.nytimes.com/2016/ 11/26/business/anger-rooms-a-smashing-new-way-to-relieve-stress.html

[35] Gustav Metzger. 1996. Damaged nature, auto-destructive art. Coracle Press, London, England.

[36] Martin Murer. 2018. Making Things Apart: Gaining Material Understanding. In Proceedings of the 2018 on Designing Interactive Systems Conference 2018 - DIS '18. ACM Press, Hong Kong, China, 497-509. https://doi.org/10.1145/3196709. 3196806

[37] Martin Murer, Verena Fuchsberger, and Manfred Tscheligi. 2015. Deconstructivist Interaction Design: Interrogating Expression and Form. Aarhus Series on Human Centered Computing 1, 1 (Oct. 2015), 4. https://doi.org/10.7146/aahcc.v1i1.21313

[38] Martin Murer, Verena Fuchsberger, and Manfred Tscheligi. 2017. Un-Crafting: DeConstructive Engagements with Interactive Artifacts. In Proceedings of the Tenth International Conference on Tangible, Embedded, and Embodied Interaction - TEI '17. ACM Press, Yokohama, Japan, 67-77. https://doi.org/10.1145/3024969.3024993

[39] Martin Murer, Anna Vallgårda, Mattias Jacobsson, and Manfred Tscheligi. 2015 Un-Crafting: Exploring Tangible Practices for Deconstruction in Interactive System Design. In Proceedings of the Ninth International Conference on Tangible, Embedded, and Embodied Interaction - TEI '14. ACM Press, Stanford, California, USA, 469-472. https://doi.org/10.1145/2677199.2683582

[40] Tuan D. Ngo, Alireza Kashani, Gabriele Imbalzano, Kate T.Q. Nguyen, and David Hui. 2018. Additive manufacturing (3D printing): A review of materials, methods, applications and challenges. Composites Part B: Engineering 143 (2018), 172 - 196. https://doi.org/10.1016/j.compositesb.2018.02.012

[41] Nouryon. 2019. Bio-based microspheres. https://expancel.nouryon.com/newsstories/bio-based-microspheres/.

[42] James Pierce and Eric Paulos. 2011. Second-hand interactions: investigating reacquisition and dispossession practices around domestic objects. In Proceedings of the 2011 annual conference on Human factors in computing systems - CHI '11. ACM Press, Vancouver, BC, Canada, 2385. https://doi.org/10.1145/1978942. 1979291

[43] James Pierce and Eric Paulos. 2015. Making Multiple Uses of the Obscura 1C Digital Camera: Reflecting on the Design, Production, Packaging and Distribution of a Counterfunctional Device. In Proceedings of the 33rd Annual ACM Conference on Human Factors in Computing Systems - CHI '15. ACM Press, Seoul, Republic of Korea, 2103-2112. https://doi.org/10.1145/2702123.2702405
[44] Julia Ringler and Holger Reckter. 2012. DESU 100: about the temptation to destroy a robot. In Proceedings of the Sixth International Conference on Tangible, Embedded and Embodied Interaction - TEI '12. ACM Press, Kingston, Ontario, Canada, 151. https://doi.org/10.1145/2148131.2148164

[45] Corina Sas, Steve Whittaker, and John Zimmerman. 2016. Design for Rituals of Letting Go: An Embodiment Perspective on Disposal Practices Informed by Grief Therapy. ACM Transactions on Computer-Human Interaction 23, 4 (Sept. 2016), 1-37. https://doi.org/10.1145/2926714

[46] A. Sydney Gladman, Elisabetta A. Matsumoto, Ralph G. Nuzzo, L. Mahadevan, and Jennifer A. Lewis. 2016. Biomimetic 4D printing. Nature Materials 15, 4 (April 2016), 413-418. https://doi.org/10.1038/nmat4544

[47] Wladyslaw Tatarkiewicz. 1972. The Great Theory of Beauty and Its Decline. The Journal of Aesthetics and Art Criticism 31, 2 (1972), 165-180. https://doi.org/10. 2307/429278

[48] Skylar Tibbits. 2014. 4D Printing: Multi-Material Shape Change. Architectural Design 84, 1 (2014), 116-121. https://doi.org/10.1002/ad.1710 arXiv:https://onlinelibrary.wiley.com/doi/pdf/10.1002/ad.1710

[49] Vasiliki Tsaknaki, Marisa Cohn, Laurens Boer, Ylva Fernaeus, and Anna Vallgårda. 2016. Things Fall Apart: Unpacking the Temporalities of Impermanence for HCI. In Proceedings of the 9th Nordic Conference on Human-Computer Interaction NordiCHI '16. ACM Press, Gothenburg, Sweden, 1-3. https://doi.org/10.1145/ 2971485.2987680

[50] Vasiliki Tsaknaki and Ylva Fernaeus. 2016. Expanding on Wabi-Sabi as a Design Resource in HCI. In Proceedings of the 2016 CHI Conference on Human Factors in Computing Systems. ACM, San Jose California USA, 5970-5983. https://doi.org/ $10.1145 / 2858036.2858459$

[51] Teunis van Manen, Shahram Janbaz, and Amir A. Zadpoor. 2017. Programming 2D/3D shape-shifting with hobbyist 3D printers. Materials Horizons 4, 6 (2017), 1064-1069. https://doi.org/10.1039/C7MH00269F

[52] Eldy S. Lazaro Vasquez and Katia Vega. 2019. From plastic to biomaterials: prototyping DIY electronics with mycelium. In Proceedings of the 2019 ACM International foint Conference on Pervasive and Ubiquitous Computing and Proceedings of the 2019 ACM International Symposium on Wearable Computers - UbiComp/ISWC '19. ACM Press, London, United Kingdom, 308-311. https://doi.org/10.1145/3341162.3343808

[53] Eldy S. Lazaro Vasquez and Katia Vega. 2019. Myco-accessories: sustainable wearables with biodegradable materials. In Proceedings of the $23 \mathrm{rd}$ International Symposium on Wearable Computers - ISWC '19. ACM Press, London, United Kingdom, 306-311. https://doi.org/10.1145/3341163.3346938

[54] Eldy S Lazaro Vasquez, Hao-Chuan Wang, and Katia Vega. 2020. The Environmental Impact of Physical Prototyping: a Five-Year CHI Review. In SelfSustainableCHI 2020. ACM, Honolulu, HI, USA, 8.

[55] Guanyun Wang, Ye Tao, Ozguc Bertug Capunaman, Humphrey Yang, and Lining Yao. 2019. A-line: 4D Printing Morphing Linear Composite Structures. In Proceedings of the 2019 CHI Conference on Human Factors in Computing Systems - CHI '19. ACM Press, Glasgow, Scotland Uk, 1-12. https://doi.org/10.1145/3290605.3300656

[56] Guanyun Wang, Humphrey Yang, Zeyu Yan, Nurcan Gecer Ulu, Ye Tao, Jianzhe Gu, Levent Burak Kara, and Lining Yao. 2018. 4DMesh: 4D Printing Morphing Non-Developable Mesh Surfaces. In The 31st Annual ACM Symposium on User Interface Software and Technology - UIST '18. ACM Press, Berlin, Germany, 623635. https://doi.org/10.1145/3242587.3242625

[57] Jingchun Wang, Zhenguo Wang, Zhengyi Song, Luquan Ren, Oingping Liu, and Lei Ren. 2019. Biomimetic Shape-Color Double-Responsive 4D Printing. Advanced Materials Technologies 4, 9 (Sept. 2019), 1900293. https://doi.org/10. 1002/admt.201900293

[58] Jennifer Weiler, Piyum Fernando, Nipuni Siyambalapitiya, and Stacey Kuznetsov. 2019. Mycelium Artifacts: Exploring Shapeable and Accessible Biofabrication. In Companion Publication of the 2019 on Designing Interactive Systems Conference 2019 Companion - DIS '19 Companion. ACM Press, San Diego, CA, USA, 69-72. https://doi.org/10.1145/3301019.3325156

[59] Shanel Wu and Laura Devendorf. 2020. Unfabricate: Designing Smart Textiles for Disassembly. In Proceedings of the 2020 CHI Conference on Human Factors in Computing Systems. ACM, Honolulu HI USA, 1-14. https://doi.org/10.1145/ 3313831.3376227

[60] Lining Yao, Jifei Ou, Chin-Yi Cheng, Helene Steiner, Wen Wang, Guanyun Wang, and Hiroshi Ishii. 2015. bioLogic: Natto Cells as Nanoactuators for Shape Changing Interfaces. In Proceedings of the 33rd Annual ACM Conference on Human Factors in Computing Systems - CHI '15. ACM Press, Seoul, Republic of Korea, 1-10. https://doi.org/10.1145/2702123.2702611

[61] ZMorph. 2020. Thick Paste Extruder. https://zmorph3d.com/products/toolheads/ thick-paste-extruder. Accessed: 2020-09-10.

[62] Amit Zoran and Leah Buechley. 2013. Hybrid Reassemblage: An Exploration of Craft, Digital Fabrication and Artifact Uniqueness. Leonardo 46, 1 (Feb. 2013), 4-10. https://doi.org/10.1162/LEON_a_00477 